\title{
MIXED CUSP FORMS AND PARABOLIC COHOMOLOGY
}

\author{
MIN HO LEE
}

(Received 24 August 1995; revised 23 February 1996)

Communicated by W. W. L. Chen

\begin{abstract}
Let $S_{k, l}(\Gamma, \omega, \chi)$ be the space of mixed cusp forms of type $(k, l)$ associated to a Fuchsian group $\Gamma$, a holomorphic map $\omega: \mathscr{H} \rightarrow \mathscr{H}$ of the upper half plane into itself and a homomorphism $\chi: \Gamma \rightarrow$ $S L(2, \mathbb{R})$ such that $\omega$ and $\chi$ are equivariant. We construct a map from $S_{k, l}(\Gamma, \omega, \chi)$ to the parabolic cohomology space of $\Gamma$ with coefficients in some $\Gamma$-module and prove that this map is injective.
\end{abstract}

1991 Mathematics subject classification (Amer. Math. Soc.): primary 11F11; secondary 11F30.

\section{Introduction}

Let $\Gamma \subset S L(2, \mathbb{R})$ be a Fuchsian group of the first kind acting on the Poincaré upper half plane $\mathscr{H}$ by linear fractional transformations. Let $\chi: \Gamma \rightarrow S L(2, \mathbb{R})$ be a homomorphism such that there is a holomorphic map $\omega: \mathscr{H} \rightarrow \mathscr{H}$ satisfying $\omega(\gamma z)=\chi(\gamma) \omega(z)$ for all $\gamma \in \Gamma$ and $z \in \mathscr{H}$. We assume that the inverse image of a parabolic subgroup of $\chi(\Gamma)$ under $\chi$ is parabolic. If $j: S L(2, \mathbb{R}) \times \mathscr{H} \rightarrow \mathbb{C}$ is the automorphy factor defined by $j\left(\left(\begin{array}{ll}a & b \\ c & d\end{array}\right), z\right)=c z+d$, then a mixed automorphic (respectively cusp) form of type $(m, n)$ is a holomorphic function $f: \mathscr{H} \rightarrow \mathbb{C}$ satisfying

$$
f(\gamma z)=j(\gamma, z)^{m} j(\chi(\gamma), \omega(z))^{n} f(z)
$$

for all $\gamma \in \Gamma$ and $z \in \mathscr{H}$ that is holomorphic (respectively vanishes) at the cusps of $\Gamma$. Certain types of mixed cusp forms can be interpreted as holomorphic forms on some families of abelian varieties (see $[4,5]$ ). Various aspects of mixed automorphic forms of the above form and their generalizations to higher dimensional cases have been investigated in a number of papers (see for example $[6,7,8,9]$ ).

This research was partially supported by a PDL award from the University of Northern Iowa.

(C) 1997 Australian Mathematical Society 0263-6115/97 \$A2.00+0.00 
A connection between the cohomology of $\Gamma$ and automorphic forms for $\Gamma$ was made by Eichler [2] and Shimura [10]. They established an isomorphism between the space of cusp forms of weight $m+2$ for $\Gamma$ and the parabolic cohomology space of $\Gamma$ with coefficients in the space of homogeneous polynomials of degree $m$ in two variables over $\mathbb{R}$. A similar isomorphism for mixed cusp forms may not be hold in general as can be seen in $[1, \S 3]$ where mixed cusp forms of type $(0,3)$ were treated in connection with elliptic surfaces. In this paper we construct a map from the space of mixed cusp forms of type $(k, l)$ associated to $\Gamma, \omega$ and $\chi$ with $k \geq 2$ to the parabolic cohomology space of $\Gamma$ with coefficients in some $\Gamma$-module and prove that this map is injective.

\section{Cocycles associated to mixed cusp forms}

In this section we review the definition of mixed cusp forms and construct a cocycle in a parabolic cohomology associated to a mixed cusp form. Let $\Gamma \subset S L(2, \mathbb{R})$ be a torsion-free Fuchsian group of the first kind acting on the Poincaré upper half plane $\mathscr{H}$. Let $\chi: \Gamma \rightarrow S L(2, \mathbb{R})$ be a homomorphism, and let $\omega: \mathscr{H} \rightarrow \mathscr{H}$ be a holomorphic map such that $\omega$ and $\chi$ are equivariant, that is,

$$
\omega(\gamma z)=\chi(\gamma) \omega(z)
$$

for all $\gamma \in \Gamma$ and $z \in \mathscr{H}$. We assume that the inverse image of a parabolic subgroup of $\Gamma^{\prime}=\chi(\Gamma)$ under $\chi$ is a parabolic subgroup of $\Gamma$ so that the $\Gamma$-cusps and $\Gamma^{\prime}$-cusps correspond. We denote by $j: S L(2, \mathbb{R}) \times \mathscr{H} \rightarrow \mathbb{C}$ the automorphy factor defined by $j(g, w)=c w+d$ if

$$
g=\left(\begin{array}{ll}
a & b \\
c & d
\end{array}\right) \in S L(2, \mathbb{R})
$$

and $w \in \mathscr{H}$.

DEFINITION 2.1. Let $m$ and $n$ be non-negative integers. A holomorphic function $f: \mathscr{H} \rightarrow \mathbb{C}$ is said to be a mixed automorphic form of type $(m, n)$ associated to $\Gamma$, $\omega$ and $\chi$ if $f$ satisfies the following conditions:

(i) $f(\gamma z)=f(z) j(\gamma, z)^{m} j(\chi(\gamma), \omega(z))^{n}$ for all $\gamma \in \Gamma$ and $z \in \mathscr{H}$.

(ii) $f$ is holomorphic at each $\Gamma$-cusp.

The function $f$ is said to be a mixed cusp form of type $(m, n)$ associated to $\Gamma, \omega$ and $\chi$ if (ii) is replaced by

(ii) ${ }^{\prime} f$ vanishes at each $\Gamma$-cusp (see [7] for details for the conditions (ii) and (ii)').

We shall denote by $S_{m, n}(\Gamma, \omega, \chi)$ the space of mixed cusp forms of type $(m, n)$ associated to $\Gamma, \omega$ and $\chi$. 
REMARK 2.2. A mixed automorphic form of type $(m, 0)$ is an automorphic form of weight $m$. On the other hand, if $\omega$ and $\chi$ are the identity maps, then a mixed automorphic form of type $(m, n)$ associated to $\Gamma, \omega$ and $\chi$ becomes a cusp form of weight $m+n$ for $\Gamma$. Mixed automorphic forms of certain types arise naturally as holomorphic forms of the highest degree on some families of abelian varieties, and various aspects of mixed automorphic forms have been investigated recently (see for example $[4,5,6,7])$. Mixed automorphic forms of several variables have also been introduced (cf. $[8,9])$.

Given a commutative ring $R$ we denote by $\mathscr{P}_{X, Y}^{n}(R)$ the space of homogeneous polynomials of degree $n$ in two variables $X$ and $Y$. Then the semigroup $M(2, R)$ of $2 \times 2$ matrices with entries in $R$ acts on $\mathscr{P}_{X, Y}^{n}(R)$ by

$$
M^{n}(\gamma) \phi(X, Y)=\phi\left({ }^{\prime}\left(\gamma^{\prime}\left(\begin{array}{l}
X \\
Y
\end{array}\right)\right)\right)=\phi\left((X, Y)^{t} \gamma^{l}\right),
$$

where $^{t}(\cdot)$ denotes the transpose of the matrix $(\cdot)$ and $\gamma^{\prime}=\operatorname{tr}(\gamma) \cdot I_{2}-\gamma=\operatorname{det}(\gamma) \gamma^{-1}$. For fixed non-negative integers $k$ and $m$ we set

$$
\mathscr{P}^{k, m}(\mathbb{C})=\mathscr{P}_{X_{1}, Y_{1}}^{k}(\mathbb{C}) \otimes \mathscr{P}_{X_{2}, Y_{2}}^{m}(\mathbb{C}),
$$

and let $\Gamma$ act on $\mathscr{P}^{k, m}(\mathbb{C})$ by $M_{\chi}^{k, m}(\gamma)=M^{k}(\gamma) \otimes M^{m}(\chi(\gamma))$, that is,

$$
M_{X}^{k, m}(\gamma)\left(\phi\left(X_{1}, Y_{1}\right) \otimes \psi\left(X_{2}, Y_{2}\right)\right)=\left(M^{k}(\gamma) \phi\left(X_{1}, Y_{1}\right)\right) \otimes\left(M^{m}(\chi(\gamma)) \psi\left(X_{2}, Y_{2}\right)\right)
$$

for all $\gamma \in \Gamma, \phi\left(X_{1}, Y_{1}\right) \in \mathscr{P}_{X_{1}, Y_{1}}^{k}(\mathbb{C})$ and $\psi\left(X_{2}, Y_{2}\right) \in \mathscr{P}_{X_{2}, Y_{2}}^{m}(\mathbb{C})$. Thus we can consider the parabolic cohomology $H_{P}^{1}\left(\Gamma, \mathscr{P}^{k, m}(\mathbb{C})\right)$ of $\Gamma$ with coefficients in $\mathscr{P}^{k, m}(\mathbb{C})$. Let $Z^{1}\left(\Gamma, \mathscr{P}^{k, m}(\mathbb{C})\right)$ be the set of 1 -cocycles for the action of $\Gamma$ on $\mathscr{P}^{k, m}(\mathbb{C})$. Thus it consists of maps $u: \Gamma \rightarrow \mathscr{P}^{k, m}(\mathbb{C})$ such that

$$
u(\gamma \delta)=u(\gamma)+M_{\chi}^{k, m}(\gamma) u(\delta)
$$

for all $\gamma, \delta \in \Gamma$. We denote by $Z_{P}^{1}\left(\Gamma, \mathscr{P}^{k, m}(\mathbb{C})\right)$ the subspace of $Z^{1}\left(\Gamma, \mathscr{P}^{k, m}(\mathbb{C})\right)$ consisting of the maps $u: \Gamma \rightarrow \mathscr{P}^{k, m}(\mathbb{C})$ satisfying

$$
u(\pi) \in\left(M_{\chi}^{k, m}(\pi)-1\right) \mathscr{P}^{k, m}(\mathbb{C})
$$

for $\pi \in P$, where $P$ is the set of parabolic elements of $\Gamma$. We also denote by $B^{1}\left(\Gamma, \mathscr{P}^{k, m}(\mathbb{C})\right)$ the set of maps $u: \Gamma \rightarrow \mathscr{P}^{k, m}(\mathbb{C})$ satisfying

$$
u(\gamma)=\left(M_{\chi}^{k, m}(\gamma)-1\right) x
$$

for all $\gamma \in \Gamma$, where $x$ is an element of $\mathscr{P}^{k, m}(\mathbb{C})$ independent of $\gamma$. Then the parabolic cohomology of $\Gamma$ with coefficients in $\mathscr{P}^{k, m}(\mathbb{C})$ is given by

$$
H_{P}^{1}\left(\Gamma, \mathscr{P}^{k, m}(\mathbb{C})\right)=Z_{P}^{1}\left(\Gamma, \mathscr{P}^{k, m}(\mathbb{C})\right) / B^{1}\left(\Gamma, \mathscr{P}^{k, m}(\mathbb{C})\right)
$$


(see for example [3, Appendix], [11, Chapter 8] for details).

Now we denote by $\Delta_{k, m}(z)$ the differential form on $\mathscr{H}$ with values in the space $\mathscr{P}^{k, m}(\mathbb{C})$ given by

$$
\Delta_{k, m}(z)=\left(X_{1}-z Y_{1}\right)^{k} \otimes\left(X_{2}-\omega(z) Y_{2}\right)^{m} d z
$$

for all $z \in \mathscr{H}$. Given a mixed automorphic form $f \in S_{k+2, m}(\Gamma, \omega, \chi)$ of type $(k+2, m)$ we also define the differential form $\Omega(f)$ on $\mathscr{H}$ by

$$
\Omega(f)=2 \pi i f(z) \Delta_{k, m}(z) .
$$

LEMMA 2.3. For each $\gamma \in \Gamma$ we have

$$
\gamma^{*} \Delta_{k, m}(z)=j(\gamma, z)^{-k-2} j(\chi(\gamma), \omega(z))^{-m}\left(M_{\chi}^{k, m}(\gamma) \Delta_{k, m}(z)\right)
$$

for all $z \in \mathscr{H}$, where $\gamma^{*} \Delta_{k, m}(z)=\Delta_{k, m}(\gamma z)$.

$$
\begin{aligned}
& \text { PROOF. Let } \gamma=\left(\begin{array}{ll}
a & b \\
c & d
\end{array}\right) \in \Gamma \subset S L(2, \mathbb{R}) \text {. Then we have } \\
& \qquad \gamma^{*} \Delta_{k, m}(z)=\left(X_{1}-(\gamma z) Y_{1}\right)^{k} \otimes\left(X_{2}-\omega(\gamma z) Y_{2}\right)^{m} d(\gamma z)
\end{aligned}
$$

for all $z \in \mathscr{H}$. But we have $d(\gamma z)=(c z+d)^{-2} d z=j(\gamma, z)^{-2} d z$ and

$$
\begin{aligned}
\left(X_{1}-(\gamma z) Y_{1}\right)^{k} & =\left(\left(X_{1}, Y_{1}\right)\left(\begin{array}{cc}
0 & 1 \\
-1 & 0
\end{array}\right)\left(\begin{array}{c}
\gamma z \\
1
\end{array}\right)\right)^{k} \\
& =\left(\left(X_{1}, Y_{1}\right)\left(\begin{array}{cc}
0 & 1 \\
-1 & 0
\end{array}\right) \gamma\left(\begin{array}{l}
z \\
1
\end{array}\right)\right)^{k}(c z+d)^{-k} \\
& =\left(\left(X_{1}, Y_{1}\right)^{k} \gamma^{k}\left(\begin{array}{cc}
0 & 1 \\
-1 & 0
\end{array}\right)\left(\begin{array}{l}
z \\
1
\end{array}\right)\right)^{k}(c z+d)^{-k} \\
& =M^{k}(\gamma)\left(X_{1}-z Y_{1}\right)^{k} j(\gamma, z)^{-k}
\end{aligned}
$$

since

$$
{ }^{\prime} \gamma^{\prime}\left(\begin{array}{cc}
0 & 1 \\
-1 & 0
\end{array}\right)={ }^{\prime} \gamma^{-1}\left(\begin{array}{cc}
0 & 1 \\
-1 & 0
\end{array}\right)=\left(\begin{array}{cc}
c & d \\
-a & -b
\end{array}\right)=\left(\begin{array}{cc}
0 & 1 \\
-1 & 0
\end{array}\right) \gamma
$$

Similarly, we have

$$
\begin{aligned}
\left(X_{2}-\omega(\gamma z) Y_{2}\right)^{m} & =\left(\left(X_{2}, Y_{2}\right)\left(\begin{array}{cc}
0 & 1 \\
-1 & 0
\end{array}\right)\left(\begin{array}{c}
\omega(\gamma z) \\
1
\end{array}\right)\right)^{m} \\
& =\left(\left(X_{2}, Y_{2}\right)\left(\begin{array}{cc}
0 & 1 \\
-1 & 0
\end{array}\right)\left(\begin{array}{c}
\chi(\gamma) \omega(z) \\
1
\end{array}\right)\right)^{m} \\
& =M^{m}(\chi(\gamma))\left(X_{2}-\omega(z) Y_{2}\right)^{m} j(\chi(\gamma), \omega(z))^{-m}
\end{aligned}
$$

hence the lemma follows. 
COROLLARY 2.4. Given a mixed cusp form $f$ in $S_{k+2, m}(\Gamma, \omega, \chi)$, we have

$$
\gamma^{*} \Omega(f)=M_{\chi}^{k, m}(\gamma) \Omega(f)
$$

for all $\gamma \in \Gamma$.

PROOF. This follows immediately from Lemma 2.3 and the transformation formula in Definition 2.1(i) for mixed automorphic forms of type $(k+2, m)$.

We fix a point $z$ in $\mathscr{H}^{*}=\mathscr{H} \cup \mathbb{Q} \cup\{\infty\}$ and for each $f \in S_{k+2, m}(\Gamma, \omega, \chi)$ we define the map $\mathscr{E}_{z}(f): \Gamma \rightarrow \mathscr{P}^{k, m}(\mathbb{R})$ by

$$
\mathscr{E}_{z}(f)(\gamma)=\int_{z}^{\gamma z} \operatorname{Re}(\Omega(f)) \in \mathscr{P}^{k, m}(\mathbb{R})
$$

for each $\gamma \in \Gamma$. Note that the integral is independent of the choice of the path joining $z$ and $\gamma z$, since $\Omega(f)$ is holomorphic. The integral is convergent even if $z$ is a cusp because of the cusp condition for the mixed cusp form $f$ given in Definition 2.1(ii)'.

PROPOSITION 2.5. For each mixed automorphic form $f \in S_{k+2, m}(\Gamma, \omega, \chi)$ the associated map $\mathscr{E}_{z}(f): \Gamma \rightarrow \mathscr{P}^{k, m}(\mathbb{C})$ is a 1-cocycle in $H_{P}^{1}\left(\Gamma, \mathscr{P}^{k, m}(\mathbb{C})\right)$ whose cohomology class is independent of the choice of the base point $z$.

PROOF. If $\gamma, \gamma^{\prime} \in \Gamma$, then we have

$$
\begin{aligned}
\mathscr{E}_{2}(f)\left(\gamma \gamma^{\prime}\right)=\int_{z}^{\gamma \gamma^{\prime} z} \operatorname{Re}(\Omega(f)) & =\int_{z}^{\gamma z} \operatorname{Re}(\Omega(f))+\int_{\gamma z}^{\gamma \gamma^{\prime} z} \operatorname{Re}(\Omega(f)) \\
& =\int_{z}^{\gamma z} \operatorname{Re}(\Omega(f))+\int_{z}^{\gamma^{\prime} z} \operatorname{Re}\left(\gamma^{*} \Omega(f)\right) .
\end{aligned}
$$

Since $\operatorname{Re}\left(\gamma^{*} \Omega(f)\right)=\operatorname{Re}\left(M_{x}^{k, m}(\gamma) \Omega(f)\right)=M_{x}^{k, m}(\gamma) \operatorname{Re}(\Omega(f))$, we obtain

$$
\mathscr{E}_{z}(f)\left(\gamma \gamma^{\prime}\right)=\mathscr{E}_{z}(f)(\gamma)+M_{x}^{k, m}(\gamma) \mathscr{E}_{z}(f)\left(\gamma^{\prime}\right) ;
$$

hence it follows that $\mathscr{E}_{z}(f)$ is a 1-cocycle for the $\Gamma$-module $\mathscr{P}^{k, m}(\mathbb{C})$. Now in order to show that it is a cocycle in the parabolic cohomology $H_{P}^{1}\left(\Gamma, \mathscr{P}^{k, m}(\mathbb{C})\right)$, let $z, z^{\prime}$ be elements of $\mathscr{H}^{*}$. Then we have

$$
\begin{aligned}
\mathscr{E}_{z^{\prime}}(f)(\gamma)-\mathscr{E}_{z}(f)(\gamma) & =\int_{z^{\prime}}^{\gamma z^{\prime}} \operatorname{Re}(\Omega(f))-\int_{z}^{\gamma z} \operatorname{Re}(\Omega(f)) \\
& =\int_{\gamma z}^{\gamma z^{\prime}} \operatorname{Re}(\Omega(f))-\int_{z}^{z^{\prime}} \operatorname{Re}(\Omega(f)) \\
& =M_{\chi}^{k, m}(\gamma) \int_{z}^{z^{\prime}} \operatorname{Re}(\Omega(f))-\int_{z}^{z^{\prime}} \operatorname{Re}(\Omega(f)) \\
& =\left(M_{\chi}^{k, m}(\gamma)-1\right) \int_{z}^{z^{\prime}} \operatorname{Re}(\Omega(f)) .
\end{aligned}
$$


If $z^{\prime}$ is a cusp $s \in \mathbb{Q} \cup\{\infty\}$ and if $\pi \in P$ is a parabolic element of $\Gamma$ fixing $s$, then $\mathscr{E}_{s}(f)(\pi)=0$, and therefore we have

$$
\mathscr{E}_{z}(f)(\pi)=-\left(M_{\chi}^{k, m}(\pi)-1\right) \int_{z}^{s} \operatorname{Re}(\Omega(f)) \in\left(M_{\chi}^{k, m}(\pi)-1\right) \mathscr{P}^{k, m}(\mathbb{C}) .
$$

Hence $\mathscr{E}_{z}(f)$ is a 1-cocycle in $H_{P}^{1}\left(\Gamma, \mathscr{P}^{k, m}(\mathbb{C})\right)$, and the proof of the proposition is complete.

\section{Pairings for mixed cusp forms}

In this section we construct a pairing on the space $S_{k+2, m}(\Gamma, \omega, \chi)$ of mixed cusp forms of type $(k+2, m)$ associated to $\Gamma, \omega$ and $\chi$. Consider the $(n+1) \times(n+1)$ integral matrix

$$
\Theta=\left((-1)^{i}\left(\begin{array}{l}
n \\
j
\end{array}\right) \delta_{n-i, j}\right),
$$

where $\delta_{n-i, j}$ is the Kronecker delta. If $(x, y) \in \mathbb{C}^{2}$, we set

$$
(x, y)^{n}=\left(x^{n}, x^{n-1} y, \ldots, x y^{n-1}, y^{n}\right) \in \mathbb{C}^{n+1} .
$$

Then we have

$$
(x, y)^{n} \Theta^{t}\left(x^{\prime}, y^{\prime}\right)^{n}=\operatorname{det}\left(\begin{array}{ll}
x & x^{\prime} \\
y & y^{\prime}
\end{array}\right)^{n}
$$

for all $(x, y),\left(x^{\prime}, y^{\prime}\right) \in \mathbb{C}^{2}$. Thus we obtain a pairing on the $n$th symmetric power $S^{n}\left(\mathbb{C}^{2}\right)$ of $\mathbb{C}^{2}$ given by

$$
\left\langle(x, y)^{n},\left(x^{\prime}, y^{\prime}\right)^{n}\right\rangle=(x, y)^{n} \Theta^{t}\left(x^{\prime}, y^{\prime}\right)^{n}=\operatorname{det}\left(\begin{array}{ll}
x & x^{\prime} \\
y & y^{\prime}
\end{array}\right)^{n} .
$$

However, the space $\mathscr{P}_{X, Y}^{n}(\mathbb{C})$ of homogenous polynomials of degree $n$ in $X$ and $Y$ can be regarded as the dual space $S^{n}\left(\mathbb{C}^{2}\right)^{*}$ of $S^{n}\left(\mathbb{C}^{2}\right)$ by identifying $X^{n-i} Y^{i}$ with $e_{1}^{\otimes(n-i)} \otimes e_{2}^{\otimes i}$ where $e_{1}=\left(\begin{array}{l}1 \\ 0\end{array}\right)$ and $e_{2}=\left(\begin{array}{l}0 \\ 1\end{array}\right)$. Thus the pairing $\langle$,$\rangle induces the pairing$ $\langle,\rangle_{X, Y}^{n}$ on $\mathscr{P}_{X, Y}^{n}(\mathbb{C})$ given by

$$
\begin{aligned}
\left\langle\sum_{i=0}^{n} a_{i} X^{n-i} Y^{i}, \sum_{j=0}^{n} b_{j} X^{n-j} Y^{j}\right\rangle_{X, Y}^{n} & =\left(a_{0}, \ldots, a_{n}\right) \Theta^{-1 t}\left(b_{0}, \ldots, b_{n}\right) \\
& =\sum_{k=0}^{n}(-1)^{n-k}\left(\begin{array}{l}
n \\
k
\end{array}\right)^{-1} a_{n-k} b_{k}
\end{aligned}
$$


(see $[3, \S 6.2]$ ). Hence we obtain a pairing of the form

$$
\left\langle\langle,\rangle=\langle,\rangle_{X_{1}, Y_{1}}^{k} \cdot\langle,\rangle_{X_{2}, Y_{2}}^{m}\right.
$$

on the space

$$
\mathscr{P}^{k, m}(\mathbb{C})=\mathscr{P}_{X_{1}, Y_{1}}^{k}(\mathbb{C}) \otimes \mathscr{P}_{X_{2}, Y_{2}}^{m}(\mathbb{C})
$$

In particular we have

$$
\begin{aligned}
\left\langle\left\langle\left( X_{1}\right.\right.\right. & \left.\left.\left.-z Y_{1}\right)^{k}\left(X_{2}-\omega(z) Y_{2}\right)^{m},\left(X_{1}-\bar{z} Y_{1}\right)^{k}\left(X_{2}-\overline{\omega(z)} Y_{2}\right)^{m}\right\rangle\right\rangle \\
& =\left\langle X_{1}-z Y_{1}, X_{1}-\bar{z} Y_{1}\right\rangle_{X_{1}, Y_{1}}^{k} \cdot\left\langle X_{2}-\omega(z) Y_{2}, X_{2}-\overline{\omega(z)} Y_{2}\right\rangle_{X_{2}, Y_{2}}^{m} \\
& =(\bar{z}-z)^{k}(\overline{\omega(z)}-\omega(z))^{m} .
\end{aligned}
$$

Let $\Theta_{1}$ (respectively $\Theta_{2}$ ) be the matrix that determines the pairing on $S^{k}\left(\mathbb{C}^{2}\right.$ ) (respectively $S^{m}\left(\mathbb{C}^{2}\right)$ ) dual to $\langle,\rangle_{X_{1}, Y_{1}}^{k}$ (respectively $\langle,\rangle_{X_{2}, Y_{2}}^{m}$ ). Now let $f$, and $g$ be mixed cusp forms in $S_{k+2, m}(\Gamma, \omega, \chi)$ so that $\operatorname{Re} \Omega(f), \operatorname{Re} \Omega(g)$ are elements of $\mathscr{P}^{k, m}(\mathbb{R}) \subset \mathscr{P}^{k, m}(\mathbb{C})$. By identifying the element

$$
\left(\sum_{i=0}^{k} a_{i} X_{1}^{k-i} Y_{1}^{i}\right) \otimes\left(\sum_{j=0}^{n} b_{j} X_{2}^{n-j} Y_{2}^{j}\right)
$$

with the vector ${ }^{\prime}\left(a_{0}, \ldots, a_{k}\right) \otimes^{t}\left(b_{0}, \ldots, b_{n}\right)$, we obtain

$$
\left\langle\langle\operatorname{Re} \Omega(f), \operatorname{Re} \Omega(g)\rangle={ }^{i} \operatorname{Re}(\Omega(f)) \wedge\left(\Theta_{1}^{-1} \otimes \Theta_{2}^{-1}\right) \operatorname{Re}(\Omega(g)) .\right.
$$

We denote the form on the right hand side of the above relation by $\Phi(f, g)$ and define the pairing $I(\cdot, \cdot): S_{k+2, m}(\Gamma, \omega, \chi) \times S_{k+2, m}(\Gamma, \omega, \chi) \rightarrow \mathbb{C}$ by

$$
I(f, g)=\int_{\Gamma \backslash \mathscr{H}} \Phi(f, g)=\int_{\Gamma \backslash \mathscr{H}}{ }^{t} \operatorname{Re}(\Omega(f)) \wedge\left(\Theta_{1}^{-1} \otimes \Theta_{2}^{-1}\right) \operatorname{Re}(\Omega(g))
$$

for $f, g \in S_{k+2, m}(\Gamma, \omega, \chi)$.

PROPOSITION 3.1. The pairing $I(\cdot, \cdot)$ on $S_{k+2, m}(\Gamma, \omega, \chi)$ is non-degenerate.

PROOF. Let $f, g \in S_{k+2, m}(\Gamma, \Omega, \chi)$. Using the relations $\operatorname{Re} \Omega(f)=\Omega(f)+\overline{\Omega(f)}$ and $\operatorname{Re} \Omega(g)=\Omega(g)+\overline{\Omega(g)}$, we obtain

$$
\Phi(f, g)=\frac{1}{4}\left({ }^{t} \Omega(f) \wedge\left(\Theta_{1}^{-1} \otimes \Theta_{2}^{-1}\right) \overline{\Omega(g)}+{ }^{t} \overline{\Omega(g)} \wedge\left(\Theta_{1}^{-1} \otimes \Theta_{2}^{-1}\right) \Omega(g)\right) .
$$


But we have

$$
\begin{aligned}
{ }^{t} \Omega(f) \wedge & \left(\Theta_{1}^{-1} \otimes \Theta_{2}^{-1}\right) \overline{\Omega(g)}=f(z) \overline{g(z)}\left\langle\left(X_{1}-z Y_{1}\right)^{k},\left(X_{1}-\bar{z} Y_{1}\right)^{k}\right\rangle_{X_{1}, Y_{1}}^{k} \\
& \times\left\langle\left(X_{2}-\omega(z) Y_{2}\right)^{m},\left.\left(X_{2}-\overline{\omega(z)} Y_{2}\right)^{m}\right|_{X_{2}, Y_{2}} ^{m} d z \wedge d \bar{z}\right. \\
= & \left.f(z) \overline{g(z)}(\bar{z}-z)^{k} \overline{(\omega(z)}-\omega(z)\right)^{m} d z \wedge d \bar{z} \\
= & (-2 i) f(z) \overline{g(z)}(\bar{z}-z)^{k}(\overline{\omega(z)}-\omega(z))^{m} d x \wedge d y \\
=( & -2 i)^{k+m+1} f(z) \overline{g(z)}(\operatorname{Im} z)^{k}(\operatorname{Im} \omega(z))^{m} d x \wedge d y .
\end{aligned}
$$

Similarly, we have

$$
\begin{aligned}
{ }^{t} \overline{\Omega(f)} \wedge\left(\Theta_{1}^{-1} \otimes \Theta_{2}^{-1}\right) \Omega(g) & =\overline{f(z)} g(z)(z-\bar{z})^{k}(\omega(z)-\overline{\omega(z)})^{m} d \bar{z} \wedge d z \\
& =(2 i)^{k+m+1} f(z) \overline{g(z)}(\operatorname{Im} z)^{k}(\operatorname{Im} \omega(z))^{m} d x \wedge d y
\end{aligned}
$$

Let $\langle,\rangle_{P}$ be the Petersson inner product on $S_{k+2, m}(\Gamma, \omega, \chi)$ given by

$$
\langle f, g\rangle_{P}=\int_{\Gamma \backslash \mathscr{H}} f(g) \overline{g(z)}(\operatorname{Im} z)^{k}(\operatorname{Im} \omega(z))^{m} d x d y
$$

(see [7, Proposition 2.1]). Then we obtain

$$
I(f, g)=\int_{\Gamma \backslash \mathscr{H}} \Phi(f, g)=-(-2 i)^{k+m-1}\left[\langle f, g\rangle_{P}+(-1)^{k+m+1}\langle g, f\rangle_{P}\right] .
$$

In particular, we have

$$
\begin{aligned}
I\left(f, i^{k+m-1} g\right) & =-(-2 i)^{k+m-1}\left[(-i)^{k+m-1}\langle f, g\rangle_{P}+(-1)^{k+m+1} i^{k+m-1}\langle g, f\rangle_{P}\right] \\
& =-2^{k+m}\left[\operatorname{Re}\langle f, g\rangle_{P}\right], \\
I\left(f, i^{k+m} g\right) & =-(-2 i)^{k+m-1}\left[(-i)^{k+m}\langle f, g\rangle_{P}+(-1)^{k+m+1} i^{k+m}\langle g, f\rangle_{P}\right] \\
& =2^{k+m} i\left[\operatorname{Im}\langle f, g\rangle_{P}\right] .
\end{aligned}
$$

Hence the non-degeneracy of the pairing $I(\cdot, \cdot)$ follows from the non-degeneracy of the Petersson inner product $\langle,\rangle_{P}$.

\section{Embeddings of mixed cusp forms into parabolic cohomology}

Let $s \in \mathbb{Q} \cup\{\infty\}$ be a cusp of $\Gamma$ such that $\sigma(\infty)=s$ with $\sigma \in S L(2, \mathbb{R})$, and let $\Gamma_{s}$ be the stabilizer of $s$ in $\Gamma$. Given $\varepsilon>0$, we set

$$
V_{s, \varepsilon}=\left\{z \in \Gamma_{s} \backslash \mathscr{H} \mid \operatorname{Im}\left(\sigma^{-1}(z)\right)^{-1}<\varepsilon\right\}
$$


We choose $\varepsilon$ such that the members of $\left\{V_{s, \varepsilon} \mid s \in \Sigma\right\}$ are mutually disjoint, where $\Sigma$ is the set of $\Gamma$-cusps. Let $S_{0}=\Gamma \backslash \mathscr{H}, S=\Gamma \backslash \mathscr{H}^{*}$, and let

$$
S_{1}=S_{0}-\bigcup_{s \in \Sigma} V_{s, \varepsilon} .
$$

As is described in $[3, \S 6.1]$, there is a triangulation $\mathscr{K}$ of $S_{1}$ satisfying the following conditions:

(i) Each element of $\Gamma$ induces a simplicial map of $\mathscr{K}$ onto itself.

(ii) For each $s \in \Sigma$ the boundary of $V_{s, \varepsilon}$ is the image of a 1-chain of $\mathscr{K}$.

(iii) There is a fundamental domain $D_{1}$ in $\mathscr{H}_{1}$ whose closure consists of finitely many simplices in $\mathscr{K}$, where $\mathscr{H}_{1}$ is the inverse image of $S_{1}$ in $\mathscr{H}$.

If $g$ denotes the genus of $S$ and if $v$ is the number of cusps of $\Gamma$, then the Fuchsian group $\Gamma$ is generated by $2 g+v$ elements

$$
\alpha_{1}, \ldots, \alpha_{g}, \beta_{1}, \ldots, \beta_{g}, \pi_{1}, \ldots, \pi_{\nu}
$$

with the relation

$$
\left(\prod_{s \in \Sigma} \pi_{s}\right) \alpha_{1} \beta_{1} \alpha_{1}^{-1} \beta_{1}^{-1} \cdots \alpha_{g} \beta_{g} \alpha_{g}^{-1} \beta_{g}^{-1}=1 .
$$

Then the boundary $\partial D_{1}$ of the fundamental domain $D_{1}$ of $S_{1}$ is given by

$$
\partial D_{1}=\sum_{s \in \Sigma} t_{s}+\sum_{i=1}^{g}\left[\left(\alpha_{i}-1\right) s_{\alpha_{i}}+\left(\beta_{i}-1\right) s_{\beta_{i}}\right],
$$

where $s_{\alpha_{i}}, s_{\beta_{i}}, t_{s}$ denote the faces of $D_{1}$ corresponding to $\alpha_{i}, \beta_{i}, \pi_{s}$, respectively.

THEOREM 4.1. Given $z \in \mathscr{H}$ and $f \in S_{k+2, m}(\Gamma, \omega, \chi)$, let $\mathscr{E}_{z}(f): \Gamma \rightarrow \mathscr{P}^{k, m}(\mathbb{R})$ be as in Section 2. Then the associated map $\mathscr{E}_{z}: S_{k+2, m}(\Gamma, \omega, \chi) \rightarrow H_{P}^{1}\left(\Gamma, \mathscr{P}^{k, m}(\mathbb{C})\right)$ is injective.

PROOF. Since the pairing $I(\cdot, \cdot)$ on $S_{k+2, m}(\Gamma, \omega, \chi)$ is non-degenerate by Proposition 3.1, in order to establish the injectivity of $\mathscr{E}_{z}$ it suffices to show that, if

$$
\mathscr{E}_{z}(f)=0 \in H_{P}^{1}\left(\Gamma, \mathscr{P}^{k, m}(\mathbb{C})\right),
$$

then $I(f, g)=0$ for all $g \in S_{k+2, m}(\Gamma, \omega, \chi)$. Thus suppose that $\mathscr{E}_{z}(f)$ is a zero cohomology class in $H_{P}^{1}\left(\Gamma, \mathscr{P}^{k, m}(\mathbb{C})\right)$. Then there is an element $C \in \mathscr{P}^{k, m}(\mathbb{R})$ such that

$$
\mathscr{E}_{z}(f)(\gamma)=\left(M_{\chi}^{k, m}(\gamma)-1\right) C
$$

for all $\gamma \in \Gamma$. We define a map $F: \mathscr{H} \rightarrow \mathscr{P}^{k, m}(\mathbb{R})$ by

$$
F(w)=\int_{z}^{w} \operatorname{Re}(\Omega(f))-C
$$


for all $w \in \mathscr{H}$. Then we have

$$
\begin{aligned}
F(\gamma w)=\int_{z}^{\gamma w} \operatorname{Re}(\Omega(f))-C & =\int_{\gamma z}^{\gamma w} \operatorname{Re}(\Omega(f))+\int_{z}^{\gamma z} \operatorname{Re}(\Omega(f))-C \\
& =\int_{z}^{w} \gamma^{*} \operatorname{Re}(\Omega(f))+\mathscr{E}_{z}(f)(\gamma)-C \\
& =M_{x}^{k, m}(\gamma)(F(w)+C)+\mathscr{E}_{z}(f)(\gamma)-C \\
& =M_{\chi}^{k, m}(\gamma) F(w)+\mathscr{E}_{z}(f)(\gamma)-\left(M_{\chi}^{k, m}(\gamma)-1\right) C \\
& =M_{\chi}^{k, m}(\gamma) F(w)
\end{aligned}
$$

for all $\gamma \in \Gamma$ and $w \in \mathscr{H}$. On the other hand, we have $d F=\operatorname{Re} \Omega(f)$. Let $g \in S_{k+2, m}(\Gamma, \omega, \chi)$, and set

$$
G(w)=\int_{z}^{w} \operatorname{Re}(\Omega(g))
$$

for all $w \in \mathscr{H}$. Then we have $d G=\operatorname{Re} \Omega(g)$ and

$$
\begin{aligned}
\Phi(f, g) & ={ }^{t} d F \wedge\left(\Theta_{1}^{-1} \otimes \Theta_{2}^{-1}\right) d G \\
& =d\left[{ }^{t} F \cdot\left(\Theta_{1}^{-1} \otimes \Theta_{2}^{-1}\right) d G\right] \\
& =d\left[{ }^{t} F \cdot\left(\Theta_{1}^{-1} \otimes \Theta_{2}^{-1}\right) \operatorname{Re}(\Omega(g))\right]
\end{aligned}
$$

If $S_{0}=\Gamma \backslash \mathscr{H}, S=\Gamma \backslash \mathscr{H}^{*}$ and $S_{1}=S_{0}-\bigcup V_{s, \varepsilon}$ as before, then we obtain

$$
\begin{aligned}
I(f, g) & =\lim _{S_{1} \rightarrow S} \int_{S_{0}} d\left[{ }^{t} F \cdot\left(\Theta_{1}^{-1} \otimes \Theta_{2}^{-1}\right) \operatorname{Re}(\Omega(g))\right] \\
& =\lim _{S_{1} \rightarrow S} \int_{\partial D_{1}}{ }^{t} F \cdot\left(\Theta_{1}^{-1} \otimes \Theta_{2}^{-1}\right) \operatorname{Re}(\Omega(g)) .
\end{aligned}
$$

However, since $F(\gamma w)=M_{x}^{k, m}(\gamma) F(w)$ and $\gamma^{*} \Omega(f)=M_{x}^{k, m}(\gamma) \Omega(f)$ for all $\gamma \in \Gamma$, for each simplex $\Xi$ and $\gamma \in \Gamma$ we have

$$
\begin{aligned}
\int_{\gamma \Xi}{ }^{t} F & \cdot\left(\Theta_{1}^{-1} \otimes \Theta_{2}^{-1}\right) \operatorname{Re}(\Omega(g)) \\
& =\int_{\Xi}{ }^{t}\left(\gamma^{*} F\right) \cdot\left(\Theta_{1}^{-1} \otimes \Theta_{2}^{-1}\right) \gamma^{*} \operatorname{Re}(\Omega(g)) \\
& =\int_{\Xi}{ }^{t} F^{t} M_{\chi}^{k, m}(\gamma) \cdot\left(\Theta_{1}^{-1} \otimes \Theta_{2}^{-1}\right) M_{\chi}^{k, m}(\gamma) \operatorname{Re}(\Omega(g)) \\
& =\int_{\Xi}{ }^{t} F \cdot\left(\Theta_{1}^{-1} \otimes \Theta_{2}^{-1}\right) \operatorname{Re}(\Omega(g)) .
\end{aligned}
$$


Hence the integral of ${ }^{t} F \cdot\left(\Theta_{1}^{-1} \otimes \Theta_{2}^{-1}\right) \operatorname{Re}(\Omega(g))$ over $\left(\alpha_{i}-1\right) s_{\alpha_{i}}+\left(\beta_{i}-1\right) s_{\beta_{i}}$ is zero for $1 \leq i \leq g$, and therefore we have

$$
I(f, g)=\sum_{s \in \Sigma} \lim _{S_{1} \rightarrow S} \int_{t_{s}}{ }^{t} F \cdot\left(\Theta_{1}^{-1} \otimes \Theta_{2}^{-1}\right) \operatorname{Re}(\Omega(g)) .
$$

Since $F(w)$ is bounded near the cusps and $\operatorname{Re}(\Omega(g))$ is rapidly decreasing at each cusp of $\Gamma$, it follows that $I(f, g)=0$; hence the injectivity of the map $\mathscr{E}_{z}$ follows.

REMARK 4.2. For non-mixed cusp forms the surjectivity of the map $\mathscr{E}_{z}$ in Theorem 4.1 also follows from the Eichler-Shimura isomorphism. However, for mixed cusp forms $\mathscr{E}_{z}$ may not be surjective in general. Although in this paper we only consider mixed cusp forms of type $(l, m)$ with $l \geq 2$, it is known that $\mathscr{E}_{z}$ is not necessarily surjective for mixed cusp forms of type $(0,3)$ (see $[1, \S 3]$ ).

\section{References}

[1] D. Cox and S. Zucker, 'Intersection numbers of sections of elliptic surfaces', Invent. Math. 53 (1979), 1-44.

[2] M. Eichler, 'Eine Verallgemeinerung der Abelschen Integrale', Math. Z. 67 (1957), 267-298.

[3] H. Hida, Elementary theory of L-functions and Eisenstein series (Cambridge Univ. Press, Cambridge, 1993).

[4] B. Hunt and W. Meyer, 'Mixed automorphic forms and invariants of elliptic surfaces', Math. Ann. 271 (1985), 53-80.

[5] M. H. Lee, 'Mixed cusp forms and holomorphic forms on elliptic varieties', Pacific J. Math. 132 (1988), 363-370.

[6] _ _ 'Periods of mixed cusp forms', Manuscripta Math. 73 (1991), 163-177.

[7] — ' 'Mixed cusp forms and Poincaré series', Rocky Mountain J. Math. 23 (1993), 1009-1022.

[8] — _ 'Mixed Siegel modular forms and Kuga fiber varieties', Illinois J. Math. 38 (1994), 692 700.

[9] — 'Mixed automorphic vector bundles on Shimura varieties', Pacific J. Math. 173 (1996), $105-226$.

[10] G. Shimura, 'Sur les intégrales attachés aux formes automorphes', J. Math. Soc. Japan 11 (1959), 291-311.

[11] - Arithmetic theory of automorphic functions (Princeton Univ. Press, Princeton, 1971).

Department of Mathematics

University of Northern Iowa

Cedar Falls

Iowa 50614

USA

e-mail: lee@math.uni.edu 